\title{
AN UNUSUAL CASE OF BIVENTRICULAR HYPERTROPHY: A RARE ADULTHOOD DIAGNOSIS
}

\author{
Rosa Lillo ${ }^{1}$, Angelica Bibiana Delogu${ }^{1}$, Gessica Ingrasciotta ${ }^{1}$, Gianluigi Perri², Maria \\ Grandinetti $^{1}$, Riccardo Marano ${ }^{1}$, Giuseppe Rovere ${ }^{1}$, and Francesca Graziani ${ }^{1}$ \\ ${ }^{1}$ Fondazione Policlinico Universitario Agostino Gemelli IRCCS \\ ${ }^{2}$ Ospedale Pediatrico Bambino Gesu
}

November 2, 2021

\begin{abstract}
A woman complaining of dyspnea and chest pain since childhood, was referred to our hospital with an initial diagnosis of biventricular hypertrophic cardiomyopathy. Multimodality imaging evaluation revealed massive right ventricular (RV) hypertrophy and severe RV outflow tract obstruction, with a final diagnosis of double chambered RV associated with small ventricular septal defect with right-to-left shunt and right partial anomalous pulmonary vein return. This represents an uncommon combination of congenital abnormalities, extremely rarely diagnosed in adulthood.
\end{abstract}

\section{AN UNUSUAL CASE OF BIVENTRICULAR HYPERTROPHY: A RARE ADULTHOOD DIAGNOSIS}

Rosa Lillo ${ }^{1,2}$, MD; Angelica Bibiana Delogu ${ }^{3}, \mathrm{MD}$; Gessica Ingrasciotta, $\mathrm{MD}^{2,4}$; Gianluigi Perri ${ }^{5}$, MD; Maria Grandinetti $^{4}$, MD; Riccardo Marano ${ }^{6}, \mathrm{MD}$; Giuseppe Rovere ${ }^{6}$, MD; Francesca Graziani ${ }^{4^{*}}, \mathrm{MD}, \mathrm{PhD}$

${ }^{1}$ Department of Emergency Medicine, Fondazione Policlinico Universitario A. Gemelli IRCCS, Rome, Italy

${ }^{2}$ Catholic University of the Sacred Heart, Rome, Italy

${ }^{3}$ Department of Pediatrics, Pediatric Cardiology Unit, Catholic University of the Sacred Heart, Rome, Italy

${ }^{4}$ Department of Cardiovascular and Thoracic Sciences, Fondazione Policlinico Universitario A. Gemelli IRCCS, Rome, Italy

${ }^{5}$ Department of Pediatric Cardiology and Cardiac Surgery, Bambino Gesù Children's Hospital IRCCS, Rome, Italy

${ }^{6}$ Department of Radiological and Hematological Sciences Section of Radiology Catholic University of Rome "A. Gemelli" University Polyclinic Foundation IRCCS, Italy

Running title : UNUSUAL CASE OF BIVENTRICULAR HYPERTROPHY

*Francesca Graziani, MD, PhD Corresponding Author Department of Cardiovascular and Thoracic Sciences, Fondazione Policlinico Universitario A. Gemelli IRCCS, Largo A. Gemelli 8, 00168 Rome, ITALY Fax number: 39-06-3055535; telephone number: 39-06-30154187; E-mail address:francesca.graziani@policlinicogemelli.it 
ABSTRACT A woman complaining of dyspnea and chest pain since childhood, was referred to our hospital with an initial diagnosis of biventricular hypertrophic cardiomyopathy. Multimodality imaging evaluation revealed massive right ventricular (RV) hypertrophy and severe RV outflow tract obstruction, with a final diagnosis of double chambered RV associated with small ventricular septal defect with right-to-left shunt and right partial anomalous pulmonary vein return. This represents an uncommon combination of congenital abnormalities, extremely rarely diagnosed in adulthood.

Key words : Congenital Heart Disease; Heart Failure; Hypertrophy; Prognosis; Imaging. CASE

Careful clinic and multimodality imaging assessment are necessary to formulate the right diagnosis and avoid harmful diagnostic delay in congenital heart disease. In our case a woman initially referred to us as "biventricular hypertrophic cardiomyopathy" received a final diagnosis of double chambered right ventricle associated with ventricular septal defect and right partial anomalous pulmonary vein return: an extremely rare combination of congenital abnormalities diagnosed in adulthood. A 23-year-old woman refugee from Nigeria was admitted to a peripheral Italian hospital for dyspnea and chest pain associated to raised troponin and she was transferred to our Intensive Care Unit with a diagnosis of biventricular hypertrophic cardiomyopathy (HCM). She reported no relevant past medical history, no family history of cardiomyopathy or sudden cardiac death. She complained of chest pain and shortness of breath from childhood and she reported several hospital admissions in her native country but with no clear diagnosis. Physical examination revealed nail clubbing and reduced physical development for her age; blood pressure was 120/80 $\mathrm{mmHg}$; heart rate was regular at $70 \mathrm{bpm}$ and $\mathrm{O} 2$ saturation was $89 \%$ on air. A loud pansystolic $2-3 / 6$ murmur was heard at left sternal border. Blood analysis revealed haemoglobin $19.3 \mathrm{~g} / \mathrm{dl}$ and hematocrit 53.1\%. The 12-leads ECG showed sinus rhythm, left and right ventricular hypertrophy and $\mathrm{T}$ wave abnormalities (Fig.1A $)$ while chest X-ray revealed a spherically shaped heart and abnormal cardiothoracic ratio (Fig. 1B ). Two-dimensional echocardiography (Fig.2, Video 1 ) showed massive right ventricle (RV) hypertrophy (15-16 mm free wall) with anomalous muscle bundles and severe RV outflow tract obstruction (RVOTO) assessed by the degree of anatomic narrowing (RVOT diameter at infundibular level $5 \mathrm{~mm}$ ) and by Doppler interrogation of the obstructed region (maximum gradient $185 \mathrm{mmHg}$ ). Other echocardiographic findings were: ventricular septum flattening, not significant hypoplastic pulmonary valve (ring $18 \mathrm{~mm}$, pulmonary artery trunk $16-17 \mathrm{~mm}$, pulmonary branches $10-12 \mathrm{~mm}$ ), a small perimembranous ventricular septal defect (VSD) (4-5 mm) with right-to-left shunt. Moreover, a left ventricular outflow tract obstruction (LVOTO) with subaortic maximum gradient of $60 \mathrm{mmHg}$ was found, with concentric left ventricular hypertrophy (LVH). Biventricular ejection fraction was normal as well as mitral and aortic valve function; mild tricuspid insufficiency was detected. During hospitalization the patient experienced attacks of paroxysmal hypoxemia (similar to tetralogy of Fallot, "cyanotic spells") with acute fall in arterial saturation associated with chest pain and an increase in cardiomyocyte injury markers, likely related to sudden worsening of dynamic RVOTO. Coronary CT Angiography (CCTA) (Fig. 3, Video 2, Video 3 ) was performed to rule-out coronary anomalies, showing a normal origin and course of coronary arteries, the presence of a right partial anomalous pulmonary vein return (PAPVR) (right superior pulmonary vein draining into the superior vena cava), and confirming a significant thickening of the RV walls, including the RVOT. Taken together, these findings suggested the final diagnosis of double chambered right ventricle (DCRV) ${ }^{1}$ with associated small VSD and PAPVR.

The Heart Team discussed the clinical case with the participation of adult and pediatric cardiologists and cardiac surgeons with an expertise in congenital heart disease and we decided to refer the patient to a Heart Transplant Center for evaluation between high risk surgical correction and cardiac transplant. The referral to a Heart Transplant center was felt necessary in case the surgery was complicated by severe post-operative RV failure (the so-called "suicide" RV) requiring ECMO support and urgent transplantation back-up. She was evaluated by two experienced centers for congenital heart disease surgery and cardiac transplantation but she was considered not suitable for surgical correction (too high risk) nor for heart transplantation for her social condition and poor compliance to medical care. The patient was lost to our follow up and came back to our GUCH out-patient clinic 5 years later. She reported of several hospital admissions for dyspnea 
and angina and she had 3 miscarriages occurred within the first trimester of pregnancy.

This case was misleading since the patient was referred to our Center with the diagnosis of biventricular HCM. However, RV hypertrophy was predominant compared to LVH and the latter looked worse than the actual measured thickness (maximum LV wall thickness $11 \mathrm{~mm}$ ) because of the small LV cavity size, due to the ventricular interdependence and RV overload. The reduction of LV size in systole along with ventricular septal bulging contributed to LVOTO. The diagnosis of congenital heart disease instead of cardiomyopathy was supported by the fact that: 1) the primary lesion was the RVOTO, 2) the association with other congenital abnormalities (VSD, PAPVR) 3) symptoms present since childhood, with some relief with squatting, 4) clubbing and cyanosis, due to the hemodynamic consequences of the RVOTO, leading to such an elevated RV pressure that caused a right to left shunt through the VSD, rarely observed in $\mathrm{DCRV}^{2,3}$.

Our case raises some important issues: the diagnostic delay led to an evolution toward a condition no more suitable for corrective surgery (prohibitive surgical risk). The patient socio-cultural condition complicated the clinical management: despite existing legal frameworks, practical access to care for transplantation can be extremely complicated for refugees ${ }^{4}$ and these social issues are also often associated with patients' poor compliance to medical care. Moreover, cultural barriers made very difficult to get the woman deeply aware of the gravity of her condition and the prohibitive pregnancy-related risks.

\section{ACKNOWLEDGMENTS}

None.

\section{CONFLICT OF INTEREST}

None.

\section{REFERENCES}

1. Loukas M, Housman B, Blaak C, et al: Double-chambered right ventricle: a review. Cardiovasc Pathol . 2013:22:417-23.

2. Moustafa S, Patton DJ, Alvarez N, et al: Double chambered right ventricle with ventricular septal defect in adults: case series and review of the literature. J Cardiovasc Ultrasound . 2015:23:48-51.

3. Singh M, Agarwala MK, Grover A, et al: Clinical, echocardiographic, and angiographic profile of patients with double-chambered right ventricle: experience with 48 cases. Angiology . 1999:50:223-31.

4. Poulakou G, Len O, Akova M. Immigrants as donors and transplant recipients: specific considerations. Intensive Care Med . 2019:45:401-403.

\section{FIGURE LEGEND}

Figure 1 . Panel A: 12-leads ECG showing sinus rhythm (52 bpm), left and right ventricular hypertrophy and repolarization abnormalities. Panel B: Posterior-anterior Chest X-ray documenting situs solitus and levocardia with significant cardiac enlargement and abnormal cardiothoracic ratio (greater than 50\%).Figure 2 . Echocardiogram. Panel A: diastolic frame of parasternal short axis view at the level of the papillary muscles. Panel B: diastolic frame of apical 4 chamber view. Severe RV hypertrophy and small LV cavity size, due to the ventricular interdependence and RV overload, are evident. Panel C: parasternal short axis at the level of great vessels with color-Doppler twin view and measurement of the severely narrowed right ventricular outflow-tract (RVOT) diameter at infundibular level. Panel D: CW Doppler interrogation of the obstructed RVOT with maximum gradient of 185 mmHg.Figure 3 . Coronary-CT Angiography (CCTA). Panel A: Coronal oblique multi-planar reconstruction (MPR) well shows the right partial anomalous pulmonary venous return (PAPVR) with the right superior pulmonary vein draining into the superior Vena Cava (SVC) (green arrow). Panel B-C: MPR (B) and 3D (C) volume rendering (VR) of the RVOT well depict the significant infundibular stenosis (yellow arrows). Panel D: 3D VR-slab along the vertical long axis (VLA) of the RV well shows the significant hypertrophy of the RV walls $(>13 \mathrm{~mm})$, papillary muscles, and the severe obstruction of the RVOT (infundibular stenosis).

Supplementary Material: The following supplementary material is available online - Movie Clips AVI: 
Video 1. Transthoracic echocardiogram video clips. Panel A: parasternal long axis view. Panel B: short axis view at the level of the papillary muscles. Both clips show the severe RV hypertrophy with anomalous muscle bundles and ventricular septum flattening, causing small LV cavity size due to ventricular interdependence. Panel C: parasternal short axis at the level of great vessels and

color-Doppler twin views focused on RVOT with Color flow Doppler mosaic pattern consistent with severe RVOT obstruction. Panel D: parasternal short axis at the level of great vessels and color-Doppler twin views focused on VSD documenting the right-to-left shunt.

Video 2 . 3D VR video along the VLA of the RV shows the right PAPVR with the right superior pulmonary vein draining into the SVC, the right atriomegaly, hypertrophy of RV walls $(>13 \mathrm{~mm})$, papillary muscles, and the severe narrowing of RVOT (infundibular stenosis).

Video 3 . MPR video of the RVOT well shows the significant infundibular stenosis.

Figure 1

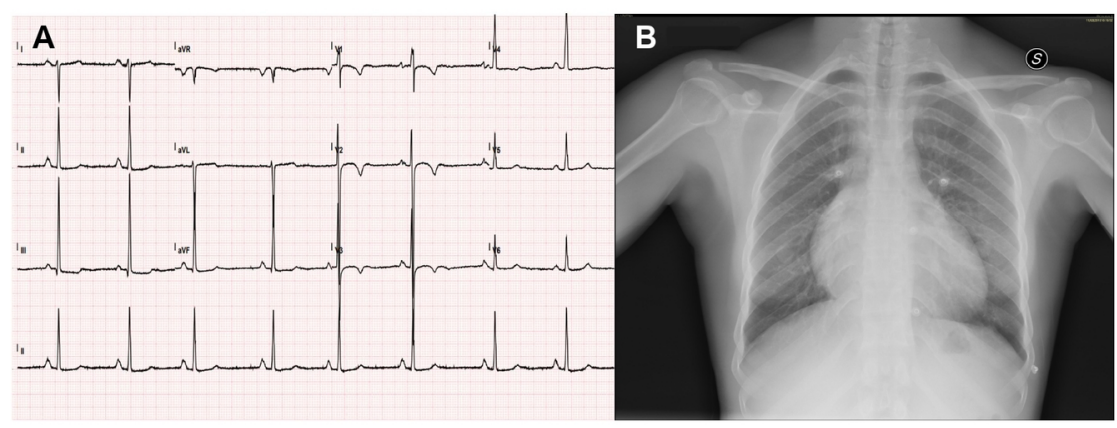



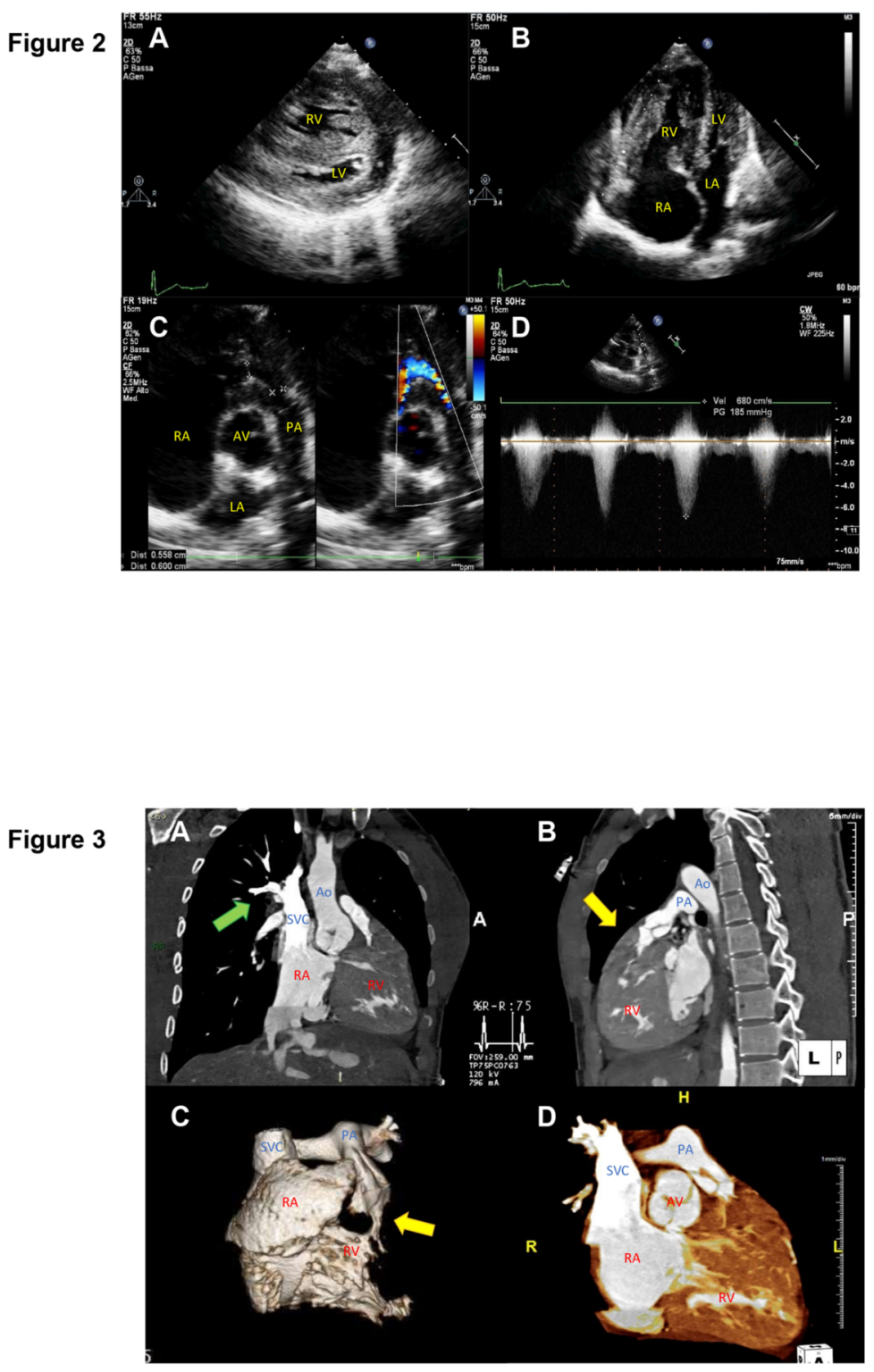